\title{
A glimmer of light for neuropsychiatric disorders
}

\author{
Steven E. Hyman ${ }^{1}$
}

\begin{abstract}
Understanding the pathogenesis of neuropsychiatric disorders is a substantial challenge for neurobiologists. It has long been hoped that identifying alleles that confer increased risk of such disorders would provide clues for neurobiological investigation. But this quest has been stymied by a lack of validated biological markers for characterizing and distinguishing the different disorders and by the genetic complexity underpinning these diseases. Now, modern genomic technologies have begun to facilitate the discovery of relevant genes.
\end{abstract}

Neuropsychiatric disorders severely compromise the well-being of those affected, with their negative effects on general health and on the ability of children to learn and of adults to work. These disorders have a relatively high prevalence ${ }^{1,2}$, can have an early onset (for example, autism in childhood and schizophrenia in young adulthood) or a relapsing-remitting course (as in mood and anxiety disorders and obsessive-compulsive disorder), and often have disabling symptoms. For these reasons, they exert, in aggregate, a devastating impact on the families involved and on the human capital of a society. To help guide countries in assessing the investment needed in the health sector, the World Health Organization (WHO) has adapted a measure of disease burden ${ }^{3}$, the Disability Adjusted Life Year (DALY), which is the sum of the years of life lost to premature mortality (YLL) in a population and the years lived with disability (YLD). Neuropsychiatric disorders, especially mood disorders, are the leading risk factors for suicide, but the major effect of these illnesses on disease burden is the disability they create. For the year 2000, the WHO estimated that neuropsychiatric disorders were responsible for $12 \%$ of all DALYs worldwide and $31 \%$ of YLDs ${ }^{4}$ (Fig. 1). For North America and Europe, where infectious disease and malnutrition are far less prominent than in the developing world, neuropsychiatric disorders were responsible for a striking $43 \%$ of all YLDs ${ }^{4}$.

Despite the disease burden attributable to neuropsychiatric disorders, and despite significant research, their mechanisms of pathogenesis and precise genetic and non-genetic risk factors have remained stubbornly out of reach. Although sustained and clever exploitation of clinical observations has produced a useful pharmacopoeia, almost all recently introduced psychotherapeutic drugs are based on reverse engineering the mechanisms of existing drugs. Arguably, no new drug targets or therapeutic mechanisms of real significance have been identified for more than four decades ${ }^{5}$. This parlous state of affairs is finally beginning to improve, in part through the application of new genomic technologies coupled to advances in neuroscience. The articles in this Insight explore some of these advances. In this Introduction, I discuss the obstacles that have delayed progress, look at some recent successes in neuropsychiatric genetics and touch on the promise of these findings for neurobiology.

\section{Challenges}

There are many reasons for the slow progress in understanding neuropsychiatric disorders. First, it is a daunting challenge to delineate the neurobiology of higher cognition, emotion regulation and executive function ('top-down' control of cognition and behaviour). Yet these are precisely the functions impaired in neuropsychiatric disorders. Second, it has been difficult to produce convincing and useful animal models of human cognitive, social and emotional functions and their dysfunction. Third, the living human brain is protected from the prying eyes and instruments of investigators by its bony skull, and for both practical and ethical reasons, human experimental neurobiology is mostly limited to indirect, non-invasive methods of investigation. Fourth, although neuropsychiatric disorders are highly influenced by genes, the genetic risk factors for common neuropsychiatric disorders have proved fiendishly complex ${ }^{6,7}$. And last, there are no well-validated objective biological markers by which to delimit precise phenotypes to use in genetics or any other type of research ${ }^{8}$. The lack of objective tests has meant that diagnosis has been made on the basis of phenomenological criteria (symptoms, signs and course of illness) selected by expert consensus. The current diagnoses listed in the fourth edition of the Diagnostic and Statistical Manual of Mental Disorders (DSM-IV) ${ }^{9}$ are useful in a rough and ready way, and indeed necessary if clinicians and researchers are to communicate, but it would be hard to argue that they provide a mirror of nature ${ }^{8}$.

\section{Genetics}

Despite these obstacles, neurobiology is making steady progress in discovering the molecular, cellular, synaptic, neural-systems, computational and cognitive underpinnings of brain function and its infirmities. The complexity of the task suggests, however, that progress will be painfully slow from the point of view of public health. Measures of genetic influence, such as heritability and recurrence risk ratio (Table 1), suggest that autism ${ }^{10}$, schizophrenia ${ }^{11,12}$ and bipolar disorder ${ }^{12}$ are among the most 'genetic' of any common medical disorder ${ }^{13}$. Many other common neuropsychiatric disorders, including major depression ${ }^{14}$, also have a significant genetic component. Therefore, it has long been hoped that alleles conferring increased disease risk could be identified. These would, in turn, provide important tools for neurobiology and would ultimately aid in developing treatments. In Alzheimer's disease, for example, the discovery of disease-causing mutations within the amyloid precursor and presenilin genes identified molecular pathways involved in pathophysiology and thus led to potential drug targets ${ }^{15}$. Spatial and temporal patterns of expression of risk-conferring genes can identify cells and circuits involved in illness and the timing of disease onset; antibodies raised against the protein products of implicated genes can 
be used to probe pathogenesis; and risk-conferring genes can be used to construct mouse models of disease.

Over the past two decades, however, efforts to identify risk-conferring alleles for the common forms of neuropsychiatric disorder have largely been unrewarding. Despite the significant role for genes highlighted by aggregate measures of their influence (Table 1), the underlying genetics of common neuropsychiatric disorders has proved highly complex, as attested by unpredictable patterns of segregation in families, lack of Mendelian ratios in twin studies and serious difficulties in replicating genetic linkage studies. There is much evidence that similar neuropsychiatric symptoms can result from different combinations of genetic risk factors $^{7}$. Conversely, the same genetic variant might be associated with multiple DSM-IV diagnoses ${ }^{7,9}$. For example, a balanced translocation discovered in a large Scottish family disrupts two genes on chromosome 1: disrupted in schizophrenia 1 (DISC1) and (DISC2 $)^{16}$. DISC1 has become a well-studied positional candidate for risk of neuropsychiatric disorders, but even within the index family, the original translocation is found in individuals with different DSM-IV diagnoses: schizophrenia, schizo-affective disorder and recurrent major depression ${ }^{16}$. Other forms of sequence variation within DISC1 have subsequently been associated with schizophrenia, schizo-affective disorder, bipolar disorder, major depression and both broad and narrow diagnoses of autism ${ }^{17}$.

At one extreme, heterogeneous genetic pathways to shared phenotypes have been shown to result from interactions among different common genetic variants at multiple genomic loci, often described as the 'common disease-common variant hypothesis'. At the other extreme, they can result from diverse rare, highly penetrant mutations, with each individual mutation causing disease in a small number of families (as is the case for retinitis pigmentosa ${ }^{18}$ ). The variants that contribute to common diseases are typically single-nucleotide polymorphisms (SNPs) that are not individually deleterious but produce risk through interaction with variants at other loci and with non-genetic factors ${ }^{19}$.

Rare, highly penetrant disease-causing mutations are by definition deleterious, causing abnormalities by loss or gain of RNA or protein functions. Not all such mutations are fully penetrant, depending on their precise biology and, potentially, on other genetic and environmental factors (such as the avoidance of phenylalanine in phenylketonuria). The identification of rare, highly penetrant disease-causing alleles is relatively straightforward with modern technologies. When common variants produce large effects on risk, they are also fairly easily identified, as in the case of the effect of the apolipoprotein E variant allele $A P O E \varepsilon 4$ in late-onset Alzheimer's disease $\mathrm{e}^{20}$ and a variant gene encoding complement factor $\mathrm{H}$ in age-related macular degeneration ${ }^{21}$. The identification of common risk-conferring variants has, however, proved extremely challenging in most cases, because of their relatively small contribution to the disease phenotype (typically a 1.1-1.3-fold increase in risk) ${ }^{19}$. Thus, the search for common variants that contribute to the risk of common disorders, such as type 2 diabetes $^{22}$, inflammatory bowel disease and breast cancer ${ }^{23}$, has only begun to be successful with the advent of tools such as the human haplotype map of SNPs and genome-wide association studies using very large populations or meta-analyses ${ }^{19,22}$.

In some cases, individual SNPs can produce deleterious mutations, especially when they influence protein translation or messenger RNA splicing. Copy-number variation (CNV), which involves larger structural alterations in chromosomes, including deletions, insertions, duplications and more complex genomic rearrangements, is more likely to produce biological effects by disrupting one or more genes or by quantitatively altering gene expression. In addition to harbouring millions of SNPs, the human genome harbours substantial CNV, some of it occurring de novo rather than being inherited. Even when risk-conferring variants are found and validated, the task of relating them to pathophysiology or to predictive testing has only just begun. Large-scale epidemiological studies are needed to better define the relationship of gene variants to particular phenotypes. Genetic association studies identify risk-conferring alleles independently of knowledge of their biological roles, so there may be little information to guide subsequent laboratory investigations. Moreover, because of the diverse genetic, environmental and random factors that

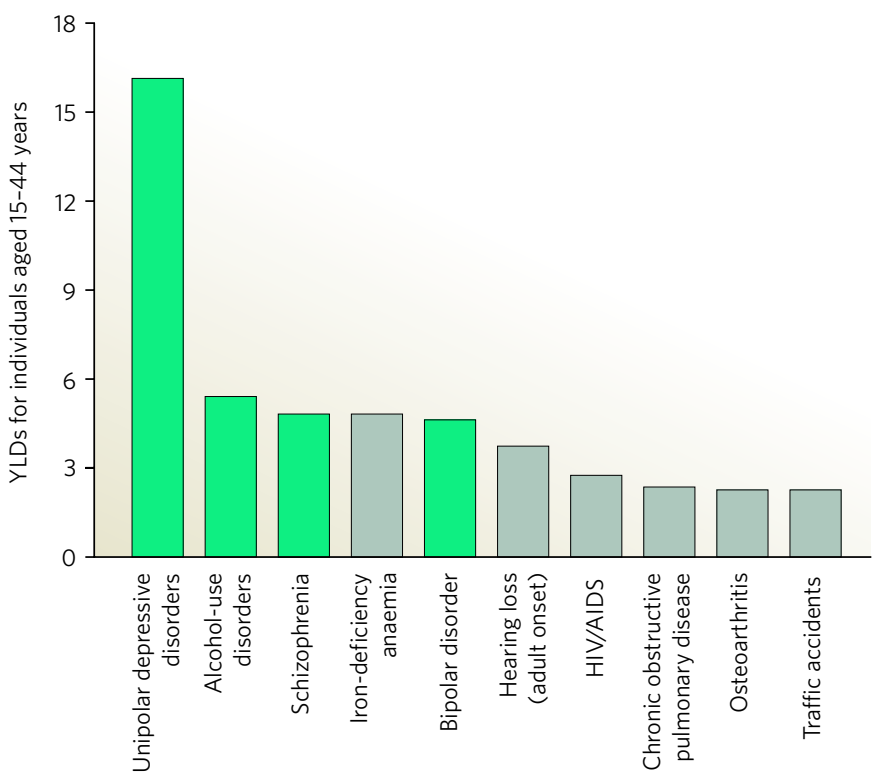

Figure 1 | Top ten causes of disability worldwide for individuals aged 15-44, as estimated for the year 2000 . The contribution of each condition is quantified as years lived with disability (YLDs). Neuropsychiatric conditions are shown in green. Data are taken from ref. 4.

lead to these common diseases, what we think of as a single 'disease' is not in a strict sense a homogeneous entity for which there is a 'Platonic' ideal phenotype. Common diseases are more likely to represent families of diseases that share major pathophysiological and symptomatic features but can differ in important characteristics such as age of onset, severity of symptoms, rate of progression, and response to treatment ${ }^{24}$.

\section{Phenotyping and clinical diagnosis}

Untangling the genetic causes of neuropsychiatric conditions has proved difficult, not only because of intrinsic genetic complexity but also because of the lack of objective diagnostic tests. Despite a decades-long search for objective markers or for intermediary neurobiological phenotypes, such as patterns of cortical thinning in schizophrenia or stress-hormone secretion in depression, validated and widely accepted markers still remain more of a goal than a reality. By contrast, even though common conditions such as type 2 diabetes and hypertension are also heterogeneous and have had shifting diagnostic criteria over time ${ }^{25}$, their diagnoses are based on objective tests that limit the heterogeneity of population samples used in research.

The DSM-IV diagnoses of conditions such as autism, schizophrenia, bipolar disorder, major depression and obsessive-compulsive disorder are not entirely without external validation: the high heritabilities of many of these disorders, and the remarkable cross-cultural similarity of symptoms, argues that the DSM-IV diagnosis is picking up a real signal. Of course, the editors of the DSM-IV did not ignore possible objective markers but had to develop diagnostic criteria in their absence. The reliance of the DSM-IV on clinical phenomenology means, however, that many patients do not fit any single diagnosis and that there are many anomalous observations, such as symptoms of schizophrenia and diverse mood disorders segregating within the same families ${ }^{7,26}$.

A complicating factor for phenotyping in genetic and neurobiological studies is that the DSM-IV classification rests on an arbitrary decision to treat all disorders as 'categorical' rather than considering some to represent quantitative deviations from normality. The DSM-IV thus posits clear boundaries between 'ill' and 'well' and between different disorders. There is, however, evidence that many neuropsychiatric disorders, such as autism, schizophrenia ${ }^{11}$ and major depression ${ }^{27}$, are better modelled quantitatively on multiple symptom dimensions, by analogy with hypertension. A consequence of the sharp boundaries drawn between 'ill' and 'well' is seen in family studies of schizophrenia: relatives of individuals 
Table 1 Relative risk of selected disorders

\begin{tabular}{llll}
\hline Disorder & $\begin{array}{l}\text { Prevalence in the } \\
\text { population (\%) }\end{array}$ & $\begin{array}{l}\text { Relative } \\
\text { risk, } \lambda_{1}{ }^{*}\end{array}$ & Heritability广 \\
\hline Autism (narrow) & 0.2 & 25 & 0.9 \\
\hline Autism (broad) & 0.7 & 25 & 0.9 \\
\hline Schizophrenia & 1.0 & 9 & $0.8-0.9$ \\
\hline Bipolar disorder & 1.0 & 8 & $0.7-0.8$ \\
\hline Major depression & 17 & $2-5$ & 0.35 \\
\hline
\end{tabular}

${ }^{*} \lambda_{1}$ is a recurrence risk ratio, in this case the fold increase in risk of developing the disorder over population base rates for a first-degree relative of an affected individual. $\uparrow$ Heritability is the proportion of phenotypic variation attributable to genetic variation.

with schizophrenia, who are considered well by DSM-IV criteria, may show cortical thinning in structural neuroimaging studies and may suffer from cognitive abnormalities ${ }^{7,11}$. A consequence of the sharp boundaries between disease categories is that many patients receive multiple DSM-IV diagnoses (described as co-morbidity), such as generalized anxiety disorder and major depression, even when the symptoms may be aetiologically related and may respond to the same treatment.

As work begins on DSM-V, the lack of objective markers remains a major problem. That said, there are proposals that might help the DSM-V fit better with scientific data. Such proposals include the addition of quantitative dimensions to the manual for some disorders, based on data derived from sources that include epidemiology, family studies and genetics. Other proposals include the possibility of updating diagnostic criteria for specific disorders without having to wait for an entirely new edition. It is probable that improvements in diagnosis will involve iterative processes in which, for example, genetic and neurobiological discoveries might suggest better disease groupings, which, in turn, might lead to additional genetic and neurobiological advances.

\section{Early progress and future directions}

Despite the challenges posed by the DSM-IV classification, as well as by a lack of objective markers for phenotyping and by genetic complexity, this year has seen significant progress in finding common variants that might contribute to bipolar disorder and schizophrenia and in finding rare, highly penetrant mutations that might give rise to schizophrenia and autism. The studies that searched for common variants contributing to bipolar disorder ${ }^{28}$ and schizophrenia ${ }^{29}$ assembled large numbers of subjects through the work of international consortia. Large sample sizes are needed because of the heterogeneity of the patients and because of the modest relative risk typically contributed by common variants. The study of bipolar disorder ${ }^{28}$ achieved significant associations with the genes $A N K 3$ and $C A C N A 1 C$ only after combining two previously published studies and adding a new one. The ANK3 protein has a role in the assembly of voltage-gated $\mathrm{Na}^{+}$channels; CACNA1C encodes the alc subunit of the L-type $\mathrm{Ca}^{2+}$ channel. Interestingly, $\mathrm{Na}^{+}$channels are targets of the anticonvulsant drugs used to treat bipolar disorder. In the study of schizophrenia ${ }^{29}$, the clearest evidence for association with a gene known as ZNF804A required a meta-analysis. Strikingly, the strength of an association with ZNF804A increased when the affected schizophrenia phenotype was extended to include bipolar disorder. If this finding holds up, it bears witness either to the pleiotropic effects of genes in neuropsychiatric disorders or to a pressing need to revise the DSM-IV with respect to its categorical split between schizophrenia and bipolar disorder, a separation that dates back to the psychiatrist Emil Kraepelin (1856-1926).

The strategy of searching for highly penetrant disease-causing mutations has received much attention in neurology but perhaps too little in psychiatry, despite the fact that single-gene disorders with autistic or psychotic symptoms have long been known. Rett syndrome ${ }^{30}$, fragile X syndrome $^{31}$ and tuberous sclerosis ${ }^{32}$ include symptoms of autism. Chromosome 22q11.2 deletions associated with DiGeorge syndrome and velocardiofacial syndrome produce psychosis in $30 \%$ of patients ${ }^{33,34}$. Historically, there had been a concern that study of these rare disorders would not shed light on the common neuropsychiatric disorders with which they shared similar symptoms. As advances in genetics have demonstrated the aetiological heterogeneity of diseases such as autism ${ }^{35}$ and schizophrenia ${ }^{7,11}$, it has become clear that useful clues should be seized wherever they arise. Just as several neurodegenerative diseases, including Alzheimer's disease ${ }^{15,36}$, Parkinson's disease ${ }^{37}$ and amyotrophic lateral sclerosis $^{38}$, can result either from multiple common variants or from rare, highly penetrant mutations, it now seems that both schizophrenia ${ }^{29,39-41}$ and autism ${ }^{35,42,43}$ show both types of genetic risk as well. Indeed a spate of recent papers has correlated excess $\mathrm{CNV}$ with risk of schizophrenia ${ }^{39-41}$; others have closed in on mutations that might cause autism by exploiting methods to identify $\mathrm{CNV}^{42,43}$. Undoubtedly, there is also a wide variety of intermediate situations in which relatively penetrant mutations act against a background of multiple disease-modifier alleles to influence age of onset or to produce milder or more severe disease.

In this Insight, Edwin Cook and Stephen Scherer describe how the widespread CNV in the human genome ${ }^{44}$ might contribute to neuropsychiatric disorders, while reminding us of the difficulty of proving that a particular mutation has a role in the disease phenotype of interest (see page 919). This point deserves to be emphasized. Biologically interesting candidate genes, in the absence of statistical demonstration of a causal role, run the risk of creating a diversion of effort with which this field is only too well acquainted. That said, appropriately validated, highly penetrant mutations provide significant opportunities for neurobiological investigation. Because common disease-related variants contribute only small increments of risk, they are likely to be highly sensitive to genetic background and environmental factors, whether their effects are being studied in people with the disease or in transgenic mouse models. Rare disease-causing mutations are more likely to produce robust phenotypes in mouse models, as they have in models of Alzheimer's disease ${ }^{45}$ and Rett syndrome ${ }^{46}$, even if they cannot be said to perfectly mimic the human disease. Understanding the biology of neuropsychiatric disorders will demand the assembly of information from multiple sources and levels of analysis.

Also in this Insight, Thomas Südhof describes the kind of compelling neurobiological analysis that is possible when penetrant mutations are identified in genes known to have significant functions in the nervous system (see page 903). The fascinating story of how mutations in the neuroligin and neurexin genes might alter the balance between excitatory and inhibitory neurotransmission is a major step forward. It is also a reminder of how far neurobiology must progress before we can understand how such mutations can produce specific deficits in social cognition. Melissa Ramocki and Huda Zoghbi focus on mutations that illuminate the importance of the regulation of gene and protein expression in the pathogenesis of neuropsychiatric disorders that begin during development (see page 912). They adduce several examples, including Rett syndrome (for which Zoghbi and colleagues were the first to identify a mutation in the MECP2 gene ${ }^{47}$ ) and fragile X syndrome, and put forward the hypothesis that loss or gain of protein and RNA functions cause remarkably overlapping cognitive and neuropsychiatric effects because of an effect on neuronal network homeostasis. In addition, Vaishnav Krishnan and Eric Nestler illustrate the sophistication required in the neurobiological investigation of what may be the most common serious neuropsychiatric disorder - depression (see page 894). They describe the utility of both genetic animal models and environmentally produced animal models.

What is the significance for neurobiology of these recent advances in neuropsychiatric genetics? Even variants that contribute a small increment of risk, as is likely to be the case for $A N K 3$ and $C A C N A 1 C^{28}$, can point to pathways that might be involved in pathophysiology and can thus suggest biological hypotheses and possible drug targets. Where highly penetrant mutations can be identified, they can provide particularly powerful tools for neurobiology, as illustrated by the neurexin and neuroligin story or the investigations into fragile $\mathrm{X}$ syndrome and Rett syndrome that have produced animal models of treatment. There is, of course, still a long distance to travel in the identification of risk-conferring alleles, of which there will be many, and in the replication of association studies and demonstration of both statistical and biological significance. There is much challenging neurobiology 
to come, but it is biology that will ultimately have enormous significance for human health. Neuropsychiatric genetics has had many wellpublicized false starts ${ }^{48}$. Perhaps not all the findings discussed here will hold up, but I feel real optimism brought about by the intelligent application of modern genomic technologies. Maybe the current glimmer of light will lead to a new dawn for the diagnosis and treatment of neuropsychiatric disorders.

1. Kessler, R. C., Chiu, W. T., Demler, O., Merikangas, K. R. \& Walters, E. E. Prevalence, severity, and comorbidity of 12-month DSM-IV disorders in the National Comorbidity Survey Replication. Arch. Gen. Psychiatry 62, 617-627 (2005).

2. Kessler, R. C. et al. Prevalence and treatment of mental disorder, 1990 to 2003. N. Engl. J. Med. 352, 2515-2513 (2005)

3. Murray, C. J. \& Lopez, A. D. The Global Burden of Disease (Harvard Univ. Press, 1996).

4. World Health Organization. World Health Report 2001 (WHO, 2001).

5. Nestler, E. J., Hyman, S. E. \& Malenka, R. J. Molecular Neuropharmacology: Foundation for Clinical Neuroscience 2nd edn (McGraw-Hill, 2008).

6. Kendler, K. S., Davis, C. G. \& Kessler, R. C. The familial aggregation of common psychiatric and substance use disorders in the National Comorbidity Survey: a family history study. Br. J. Psychiatry 170, 541-548 (1997)

7. Craddock, N., O'Donovan, M. C. \& Owen, M. J. The genetics of schizophrenia and bipolar disorder: dissecting psychosis. J. Med. Genet. 42, 193-204 (2005).

8. Hyman, S. E. Can neuroscience be integrated into the DSM-V? Nature Rev. Neurosci. 8 725-732 (2007).

9. American Psychiatric Association. Diagnostic and Statistical Manual of Mental Disorders 4th edn, text revision (American Psychiatric Association, 2000).

10. Freitag, C. M. The genetics of autistic disorders and its clinical relevance: a review of the literature. Mol. Psychiatry 12, 2-22 (2007)

11. Sullivan, P. F., Kendler, K. S. \& Neale, M. C. Schizophrenia as a complex trait: evidence from a meta-analysis of twin studies. Arch. Gen. Psychiatry 60, 1187-1192 (2003).

12. Merikangas, K. R. \& Risch, N. Will the genomics revolution revolutionize psychiatry? Am. J. Psychiatry 160, 625-635 (2003).

13. Merikangas, K. R. \& Risch, N. Genomic priorities and public health. Science 302, 599-601 (2003).

14. Kendler, K. S., Gatz, M., Gardner, C. O. \& Pederson, N. L. A Swedish national twin study of lifetime major depression. Am. J. Psychiatry 163, 109-114, (2006).

15. Hardy, J. A hundred years of Alzheimer's disease research. Neuron 52, 3-13 (2006).

16. St. Clair, D. et al. Association within a family of a balanced autosomal translocation with major mental illness. Lancet 336, 13-16 (1990).

17. Hennah, W. et al. DISC1 association, heterogeneity and interplay in schizophrenia and bipolar disorder. Mol. Psychiatry advance online publication, doi:10.1038/mp.2008.22 (4 March 2008).

18. Pacione, L. R., Szego, M. J., Ikeda, S., Nishina, P. M. \& Mclnnes, R. R. Progress toward understanding the genetic and biochemical mechanisms of inherited photoreceptor degenerations. Annu. Rev. Neurosci. 26, 657-700 (2003).

19. Hunter, D. J., Altshuler, D. \& Rader, D.J. From Darwin's finches to canaries in the coal mine - mining the genome for new biology. N. Engl. J. Med. 358, 2760-2763 (2008).

20. Strittmatter, W. J. et al. Apoliprotein E: high avidity binding to $\beta$-amyloid and increased frequency of type 4 allele in late-onset familial Alzheimer disease. Proc. Natl Acad. Sci. USA 90, 1977-1981 (1993)

21. Edwards, A. O. et al. Complement factor $\mathrm{H}$ variant increases the risk of age-related macular degeneration. Science 308, 419-421 (2005).

22. Zeggini, E. et al. Meta-analysis of genome-wide association data and large-scale replication identifies additional susceptibility loci for type 2 diabetes. Nature Genet. 40, 638-645 (2008).
23. Easton, D. F. et al. Genome-wide association study identifies novel breast cancer susceptibility loci. Nature 447, 1087-1093 (2007).

24. Ciardiello, F. \& Tortora, G. EGFR antagonists in cancer treatment. N. Engl. J. Med. 358, 1160-1174 (2008)

25. Chobanian, A. V. et al. National High Blood Pressure Education Program Coordinating Committee. Seventh report of the Joint National Committee on Prevention, Detection, Evaluation, and Treatment of High Blood Pressure. Hypertension 42, 1206-1252 (2003)

26. Millar, J. K. et al. Disruption of two novel genes by a translocation co-segregating with schizophrenia. Hum. Mol. Gen. 9, 1415-1423 (2000)

27. Kendler, K. S. \& Gardner, C. O. Jr. Boundaries of major depression: an evaluation of DSM-IV criteria. Am. J. Psychiatry 155, 172-177 (1998).

28. Ferreira, M. A. R. et al. Collaborative genome-wide association analysis supports a role for ANK3 and CACNA1C in bipolar disorder. Nature Genet. 40, 1056-1058 (2008).

29. O'Donovan, M. C. et al. Identification of loci associated with schizophrenia by genomewide association and follow-up. Nature Genet. 40, 1053-1055 (2008).

30. Chahrour, M. \& Zoghbi, H. Y. The story of Rett syndrome: from clinic to neurobiology. Neuron 56, 422-437 (2007)

31. Reddy, K. S. Cytogenetic abnormalities and fragile-X syndrome in autism spectrum disorders. BMC Med. Genet. 6, 3-19 (2005)

32. Lewis, J., Thomas, H., Murphy, K. \& Sampson, J. Genotype and psychological phenotype in tuberous sclerosis. J. Med. Genet. 41, 203-207 (2004).

33. Williams, N. M. O'Donovan, M. C. \& Owen, M. J. Chromosome 22 deletion syndrome and schizophrenia. Int. Rev. Neurobiol. 73, 1-27 (2006).

34. Kobrynski, L. J. \& Sullivan, K. E. Velocardiofacial syndrome, DiGeorge syndrome: the chromosome 22q11.2 deletion syndromes. Lancet 370, 1443-1452 (2007).

35. Veenstra-Vanderweele, J., Christian, S. L. \& Cook, E. H. Jr. Autism as a paradigmatic complex genetic disorder. Annu. Rev. Genomics Hum. Genet. 5, 379-405 (2004).

36. Waring, S. C. \& Rosenberg, R. N. Genome-wide association studies in Alzheimer disease. Arch. Neurol. 65, 329-334 (2008).

37. Farrer, M. J. Genetics of Parkinson disease: paradigm shifts and future prospects. Nature Rev. Genet. 7, 306-318 (2006)

38. Valdmanis, P. N. \& Rouleau, G. A. Genetics of familial amyotrophic lateral sclerosis. Neurology 70, 144-152 (2008).

39. Walsh, T. et al. Rare structural variants disrupt multiple genes in neurodevelopmental pathways in schizophrenia. Science 320, 539-543 (2008).

40. $\mathrm{Xu}, \mathrm{B}$. et al. Strong association of de novo copy number mutations with sporadic schizophrenia. Nature Genet. 40, 880-885 (2008).

41. The International Schizophrenia Consortium. Rare chromosomal deletions and duplications increase risk of schizophrenia. Nature 455, 237-241 (2008).

42. Weiss, L. A. et al. Association between microdeletion and microduplication at $16 \mathrm{p} 11.2$ and autism. N. Engl. J. Med. 358, 667-675 (2008)

43. Morrow, E. M. et al. Identifying autism loci and genes by tracing recent shared ancestry. Science 321, 218-223 (2008).

44. Redon, R. et al. Global variation in copy number in the human genome. Nature $\mathbf{4 4 4}$ 444-454 (2006).

45. Gotz, J. \& Ittner, L. M. Animal models of Alzheimer's disease and frontotemproal dementia. Nature Rev. Neurosci. 9, 532-544 (2008).

46. Watase, K. \& Zoghbi, H. Y. Modelling brain diseases in mice: the challenges of design and analysis. Nature Rev. Genet. 4, 296-307 (2003)

47. Amir, R. E. et al. Rett syndrome is caused by mutations in X-linked MECP2, encoding methyl-CpG binding protein 2. Nature Genet. 23, 185-188 (1999).

48. Egeland, J. A. et al. Bipolar affective disorders linked to DNA markers on chromosome 11. Nature 325, 783-787 (1987)

Author Information Reprints and permissions information is available at wWW.nature.com/reprints. The author declares no competing financial interests. Correspondence should be addressed to the author (seh@harvard.edu). 